\title{
Utilidad del análisis por medio de la razón de cambio entre el diferencial de la temperatura cortical cerebral y la cronicidad de la epilepsia
}

\author{
Usefulness of the analysis by means of the rate of \\ change between the differential of cerebral cortical \\ temperature and the chronicity of epilepsy
}

Enrique de Font-Réaulx, ${ }^{*}$ Miguel Ángel Collado-Corona, ${ }^{\ddagger}$ Emilio Arch-Tirado, $\$$ Javier Terrazo-Lluch, ${ }^{\star}$ Ramón López-López," Paul Shkurovich-Bialik, ${ }^{\ddagger}$ Luis Guillermo Díaz-López," Ernesto Ramírez-Navarrete, ${ }^{* *}$ Adalberto González-Astiazarán ${ }^{\star *}$

Citar como: de Font-Réaulx E, Collado-Corona MÁ, Arch-Tirado E, Terrazo-Lluch J, López-López R, Shkurovich-Bialik P et al. Utilidad del análisis por medio de la razón de cambio entre el diferencial de la temperatura cortical cerebral y la cronicidad de la epilepsia. An Med (Mex). 2020; 65 (3): 182-186. https://dx.doi.org/10.35366/95674

\section{RESUMEN}

Introducción: En la actualidad, la epilepsia afecta aproximadamente a 60 millones de personas de todas las edades, aunque su frecuencia es mayor en niños y mayores de 60 años. El cuadro clínico de la misma es multifactorial y variado, lo que hace necesario para el clínico contar con modelos matemáticos que midan de manera objetiva el comportamiento, la interrelación y la asociación que pudiesen estar presentes o no de dos o más variables. Este artículo propone un análisis matemático de apoyo al epileptólogo. Objetivo: Evaluar la utilidad del análisis por medio de la razón de cambio entre el diferencial de la temperatura cortical cerebral y la cronicidad de la epilepsia. Material y métodos: Por medio de un estudio prospectivo/

\section{ABSTRACT}

Introduction: Epilepsy currently affects approximately 60 million people of all ages, although its frequency is higher in children and over 60 years. The clinical picture of it is multifactorial and varied, which makes it necessary for the clinician to have mathematical models that objectively measure the behavior, interrelation and association that may or may not be present of two or more variables. This article proposes a mathematical analysis to support the epileptologist. Objective: To evaluate the usefulness of the analysis by means of the ratio of change between the differential in cerebral cortical temperature and the chronicity of epilepsy. Material and methods: Through a descriptive-prospective study, 12 patients
* Neurocirugía y Clínica de Epilepsia. Centro Neurológico del Centro Médico ABC.

₹ Neurofisiología Clínica y Clínica de Epilepsia. Centro Neurológico del Centro Médico ABC.

$\S$ Investigación, Centro Neurológico, Centro Médico ABC.

"Neurocirugía, Centro Médico «La Raza», Instituto Mexicano del Seguro Social.

I Neuroanestesiología, Instituto Nacional de Neurología y Neurocirugía, Ciudad de México.

** Neuropediatría y Clínica de Epilepsia, Centro Neurológico del Centro Médico ABC.

Recibido para publicación: 30/05/2020. Aceptado: 10/08/2020.
Correspondencia:

Enrique de Font-Réaulx

Av. Carlos Fernández Graef Núm. 154, Santa Fe, Contadero,

Cuajimalpa de Morelos, 05330, Ciudad de México, CDMX.

Tel: 5549905938

E-mail: defontreaulx@hotmail.com

Abreviaturas:

$\mathrm{ECOG}=$ Electrocorticografía .

$\mathrm{MTI}=$ Mapeo por termografía infrarroja .

MTR = Máxima temperatura registrada en el cerebro.

$\mathrm{TZI}=$ Temperatura de la zona irritativa. 
descriptivo, se estudiaron 12 pacientes que cumplieron con los criterios de inclusión del estudio. Se calcularon las diferenciales entre las temperaturas registradas en el cerebro, máxima temperatura registrada en el cerebro y la temperatura de la zona irritativa en pacientes sometidos a cirugía de epilepsia. Resultados: Observamos claramente la relación que existe entre las diferencias de las temperaturas registradas y la cronicidad de la epilepsia. Discusión: Los ángulos obtenidos demuestran que, a mayor ángulo de inclinación, también mayor es la diferencia de las temperaturas con base en la duración de la enfermedad y viceversa. Conclusión: El uso de la razón de cambio para el análisis de cuatro variables, estando dos de ellas relacionadas con el tiempo y dos con la temperatura registrada en áreas del cerebro, ayuda a la interpretación de las variables con respecto al mismo sujeto.

Palabras clave: Cirugía de epilepsia, temperatura cortical, razón de cambio.

Nivel de evidencia: III were studied. Differentials between the temperatures recorded in the brain were calculated: maximum registered temperature in the brain and the temperature of the irritative zone, in patients undergoing epilepsy surgery. Results: We clearly observe the relationship between the differences in recorded temperatures and the chronicity of epilepsy. Discussion: The angles obtained show that the greater the angle of inclination, the greater the temperature difference is based on the duration of the disease and vice versa. Conclusion: The use of the rate of change for the analysis of four variables, two of them being related to time and two to the temperature registered in areas of the brain, helps to interpret the variables with respect to the same subject.

Keywords: Epilepsy surgery, cortical temperature, rate of change.

Level of evidence: III

traumatismos craneoencefálicos, tumores cerebrales, enfermedades infecciosas, trastornos metabólicos adquiridos, enfermedades degenerativas del sistema nervioso central, abuso en ingesta de bebidas alcohólicas, tóxicos, entre otros.

Dada la complejidad y cantidad de las variables relacionadas y las posibles combinaciones de éstas, es recomendable proponer modelos matemáticos que

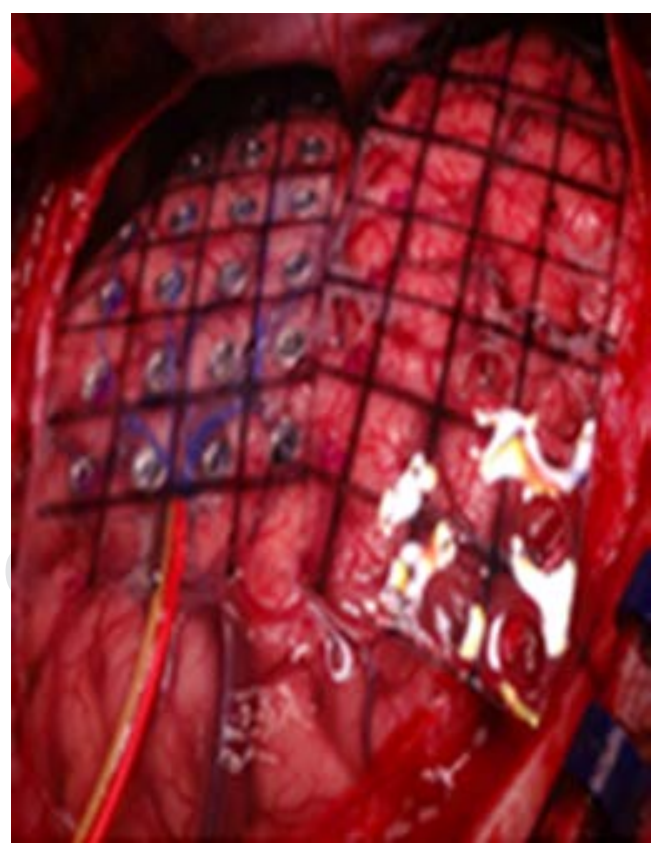

Figura 1: Cuadrícula de acetato estéril con perforaciones que coinciden con la localización de los electrodos y el tamaño de la rejilla para electrocorticografía. 
involucren el comportamiento, interrelación o, en su caso, la asociación de dos o más variables. La razón de cambio de una variable con respecto a otra determina el cambio de una con base en la unidad de cambio de la otra, lo que ayudaría de manera significativa al clínico a conocer la proporcionalidad de cuatro mediciones con respecto al tiempo.

Objetivo: evaluar la utilidad del análisis por medio de la razón de cambio entre el diferencial de la temperatura cortical cerebral y la cronicidad de la epilepsia.

\section{MATERIAL Y MÉTODOS}

Estudio observacional prospectivo. Este artículo está basado en los resultados del reporte original publicado por de Font-Réaulx y colaboradores, ${ }^{6}$ el cual fue tomado como fuente idónea para los fines de la presente propuesta; se obtuvieron los siguientes datos:

\section{Sujetos de estudio}

Se estudiaron 12 pacientes sometidos a cirugía de epilepsia farmacorresistente que fueron presentados, valorados y aprobados en forma colegiada en la Clínica de Epilepsia del Centro Neurológico del Centro Médico ABC para someterse al procedimiento quirúrgico, previo consentimiento informado y que cumplieron con los criterios de inclusión.

Se obtuvo la temperatura meníngea con un termómetro infrarrojo, rango de temperatura -50 a $330{ }^{\circ} \mathrm{C}$ $\left(-58\right.$ a $\left.626{ }^{\circ} \mathrm{F}\right)$, precisión $0-330^{\circ} \mathrm{C}\left(32-626{ }^{\circ} \mathrm{F}\right) \pm 1.5$ ${ }^{\circ} \mathrm{C}\left( \pm 2.7{ }^{\circ} \mathrm{F}\right)$, resolución $0.1^{\circ} \mathrm{C}$ o $0.1^{\circ} \mathrm{F}$, repetibilidad $1 \%$ de la lectura o $1{ }^{\circ} \mathrm{C}$, tiempo de respuesta 500 $\mathrm{ms}$, respuesta del 95\%, respuesta espectral 8-14 $\mu \mathrm{m}$, configuración de emisividad 0.95 , distancia al tamaño del punto $12: 1$, temperatura de funcionamiento $0-40{ }^{\circ} \mathrm{C}\left(32-104{ }^{\circ} \mathrm{F}\right)$, humedad de funcionamiento $10-90 \%$, humedad relativa hasta $30^{\circ} \mathrm{C}\left(86^{\circ} \mathrm{F}\right)$, temperatura de almacenamiento -20 a $60{ }^{\circ} \mathrm{C}(-4$ a 140 ${ }^{\circ} \mathrm{F}$ ), potencia $1.5 \mathrm{~V} \mathrm{AAA} * 2$ baterías. La medición de la temperatura meníngea se realiza inmediatamente al tener acceso a este plano, evitando factores que pudieran afectarla (irrigación, temperatura ambiente, distancia de registro, etcétera). Se sabe que la temperatura de una corteza cerebral expuesta puede descender entre 5 a $10{ }^{\circ} \mathrm{C}$ con respecto a la temperatura corporal.

Posteriormente, se registró con fotografías macroscópicas la corteza cerebral expuesta, según cada caso en particular, y se colocó una lámina de silicón termosensible/termocromático sobre la corteza cerebral expuesta hasta obtenZgenes térmicas portátil $\mathrm{Kmoon}^{\circledR}$ de alta resolución que combina las funciones de la temperatura de la superficie y la termografía infrarroja en tiempo real. Se utilizó un dispositivo de imagen infrarrojo profesional, de alta precisión y eficiencia, que puede convertir una imagen térmica en una imagen visible, que evalúa rápidamente los diferentes gradientes de los valores térmicos del cerebro y puede combinar imágenes visibles e infrarrojas, resolución de imagen infrarroja/imagen visible: 3,600 píxeles/0.3 megapíxeles, pantalla: pantalla de color de alta resolución de 2.4 pulgadas, resolución completa; resolución: $60 * 60$; resolución de la imagen visible: 0.3 megapíxeles, píxel total: $3,600, \mathrm{FOV} /$ longitud focal más corta: $20 \times 20 / 0.5 \mathrm{~m}$, sensibilidad térmica: $0.5{ }^{\circ} \mathrm{C}$, modo de medición: termopila, rango de temperatura: -20 a $300{ }^{\circ} \mathrm{C}\left(-4\right.$ a $\left.572{ }^{\circ} \mathrm{F}\right)$, precisión de medición: $\pm 2 \%$ o $\pm 2{ }^{\circ} \mathrm{C}\left( \pm 4{ }^{\circ} \mathrm{F}\right)$, rango de longitud de onda: 8-14 $\mu \mathrm{m}$, emisividad: 0.1-1.0 (ajustable), frecuencia de imagen: 6HZ; enfoque: modo fijo; paleta: hierro rojo, arco iris de alto contraste, escala de grises (brillo blanco) y escala de grises (brillo negro); opción de visión: 5 tipos de infrarrojo completo a la visión completa, mezcla visible e infrarroja; almacenamiento de imágenes: tarjeta SD (4G); formato de archivo: bmp; tipo de batería: batería AA de $4 * 1.5 \mathrm{~V}$; autenticación: CE, RoHS; temperatura de funcionamiento: -5 a $40{ }^{\circ} \mathrm{C}\left({ }^{\circ} \mathrm{F}\right.$ a 104$)$; humedad relativa: $10 \%$ HR a $80 \%$ HR.

Para el MTI se coloca sobre la corteza cerebral un prototipo de cuadrícula perforada de acetato estéril transparente (Figura 1) de dimensiones iguales a las de la malla de ECoG, obteniendo una concordancia precisa de cada perforación de la cuadrícula con la rejilla de ECoG en los casos que así se requiera.

Los pacientes fueron tres hombres $(25 \%)$ y nueve mujeres (75\%), el promedio de edad fue de 32.5 \pm 16.6596 años, el valor del índice de asimetría fue de 0.795 , por lo que la distribución está sesgada a la derecha, con un valor para la Kurtosis de 0.047, por lo que la distribución es plana debido al rango y tamaño de la población. Destaca el hecho de que la desviación estándar de las TZI y la MTR tienden a la igualdad, de esta manera los valores registrados con respecto a la media conllevan homocedasticidad (Tabla 1).

\section{Propuesta del modelo}

Se calcularon las diferenciales entre las temperaturas registradas en el cerebro MTR y la TZI, en pa- 
Tabla 1: Estadística descriptiva.

\begin{tabular}{|c|c|c|c|c|c|c|c|c|c|c|}
\hline & \multirow[b]{2}{*}{$\mathrm{N}$} & \multirow[b]{2}{*}{ Rango } & \multirow[b]{2}{*}{ Mínimo } & \multirow[b]{2}{*}{ Máximo } & \multicolumn{2}{|c|}{ Media } & \multirow[b]{2}{*}{$\begin{array}{l}\text { Desviación } \\
\text { estándar }\end{array}$} & \multirow[b]{2}{*}{ Varianza } & \multirow[b]{2}{*}{$\begin{array}{l}\text { Índice de } \\
\text { asimetría }\end{array}$} & \multirow[b]{2}{*}{$\begin{array}{l}\text { Kurto- } \\
\text { sis }\end{array}$} \\
\hline & & & & & & $\begin{array}{c}\text { Error } \\
\text { estándar }\end{array}$ & & & & \\
\hline Edad & 12 & 55 & 11 & 66 & 32.5 & 4.8092 & 16.6596 & 277.545 & 0.795 & 0.047 \\
\hline Duración epilepsia & 12 & 43 & 2 & 45 & 15 & 3.616 & 12.5263 & 156.909 & 1.423 & 1.997 \\
\hline Temperatura zona irritativa & 12 & 6.4 & 28.5 & 34.9 & 30.5333 & 0.5346 & 1.8519 & 3.43 & 1.241 & 1.469 \\
\hline Máxima temperatura cerebral & 12 & 4.9 & 31.7 & 36.6 & 33.9416 & 0.503 & 1.7427 & 3.037 & 0.259 & -1.198 \\
\hline Delta temperatura & 12 & 5.9 & 1.2 & 7.1 & 3.4083 & 0.4673 & 1.6188 & 2.621 & 0.958 & 1.205 \\
\hline
\end{tabular}

cientes sometidos a cirugía para el tratamiento de epilepsia farmacorresistente, se calculó el diferencial (edad del paciente en la intervención (EPI) y la edad de inicio de las crisis (EIC), el resultado obtenido se convirtió en tangente con la finalidad de analizar el cambio de estas variables en función del ángulo de inclinación resultante.

Con la finalidad de evaluar si existía alguna razón entre las variables del estudio, se analizó el cociente resultante con la diferencia entre los datos estudiados, con la finalidad de calcular el ángulo tangente y poder estimar si existía una tendencia a partir del análisis de los ángulos resultantes con la siguiente fórmula:

Tabla 2: Variables analizadas.

\begin{tabular}{cccccc}
\hline & \multicolumn{2}{c}{ Edad (años) } & & \multicolumn{2}{c}{ Temperatura $\left({ }^{\circ} \mathrm{C}\right)$} \\
\cline { 6 - 6 } \cline { 5 - 6 } Género & EPI & $\begin{array}{c}\text { Con epilepsia } \\
(\text { EIC) }\end{array}$ & & $\begin{array}{c}\text { Máxima } \\
(\text { MTR })\end{array}$ & $\begin{array}{c}\text { Zona irritativa } \\
(\mathrm{TZI})\end{array}$ \\
\hline Masculino & 47 & 20 & & 33.8 & 31.6 \\
Masculino & 22 & 9 & & 31.7 & 29.1 \\
Femenino & 22 & 7 & & 32.0 & 29.3 \\
Femenino & 13 & 8 & & 33.6 & 30.1 \\
Femenino & 38 & 31 & & 33.4 & 30.6 \\
Femenino & 26 & 2 & & 36.1 & 34.9 \\
Femenino & 11 & 11 & & 33.5 & 29.0 \\
Femenino & 29 & 19 & & 36.6 & 32.2 \\
Femenino & 32 & 10 & & 36.2 & 29.1 \\
Femenino & 56 & 45 & & 31.7 & 30.0 \\
Femenino & 28 & 16 & & 33.4 & 28.5 \\
\hline
\end{tabular}

$\mathrm{EPI}=$ edad del paciente en la intervención, EIC = edad de inicio de las crisis, MTR = máxima temperatura registrada en el cerebro, TZI = temperatura de la zona irritativa.
Fórmula 1:

$$
\tan \alpha=\frac{\text { MRT }- \text { TZI }}{\text { EPI }- \text { EIC }}
$$

También se invirtió el numerador con el denominador para evaluar el inverso con base en la temperatura registrada, analizando la inclinación de la pendiente:

Fórmula 2:

$\tan \alpha=\frac{\text { EPI }- \text { EIC }}{\text { MRT }- \text { TZI }}$

\section{Análisis de datos}

Se codificaron los datos existentes de los pacientes a causa de que el objetivo final del trabajo es la propuesta de un modelo matemático, por lo que no se calculó el tamaño de la muestra, de esta manera se agruparon y codificaron las variables que se utilizaron para calcular las razones entre las variables (Tablas 2 y 3).

En los datos se demuestra claramente la relación que existe entre las diferencias de las temperaturas registradas y la cronicidad de la epilepsia. Los ángulos obtenidos demuestran que a mayor ángulo de inclinación, mayor es la diferencia de las temperaturas con base en la duración de la enfermedad y viceversa; las diferencias de magnitudes entre el numerador y el denominador determinan la razón de cambio con respecto a las mediciones analizadas.

\section{DISCUSIÓN}

La utilización de razones aritméticas para evaluar el comportamiento de cuatro variables simultáneamen- 
Tabla 3: Análisis de datos.

\begin{tabular}{|c|c|c|c|}
\hline$\frac{\text { MRT-TZI }}{\text { EPI-EIC }}$ & $\operatorname{Tan} \alpha$ & $\frac{\text { EPI-EIC }}{\text { MRT-TZI }}$ & $\operatorname{Tan} \boldsymbol{\alpha}$ \\
\hline$\frac{22-7}{32-29.3}=\frac{15.0}{2.7}=5.5556$ & $79^{\circ} 47^{\prime}$ & $\frac{2.7}{15.0}=0.18$ & $10^{\circ} 12^{\prime}$ \\
\hline$\frac{13-8.0}{30.1-33.6}=\frac{5.0}{3.5}=1.4286$ & $55^{\circ}$ & $\frac{3.5}{5.0}=0.7$ & $34^{\circ} 59^{\prime}$ \\
\hline$\frac{47-20.0}{33.8-31.6}=\frac{27.0}{2.2}=12.2727$ & $85^{\circ} 20^{\prime}$ & $\frac{2.2}{27.0}=0.0815$ & $4^{\circ} 39^{\prime}$ \\
\hline$\frac{38-31.0}{33.4-30.6}=\frac{7.0}{2.8}=2.5$ & $68^{\circ} 11^{\prime}$ & $\frac{2.8}{7.0}=0.4$ & $21^{\circ} 48^{\prime}$ \\
\hline$\frac{22-9.0}{31.7-29.1}=\frac{13.0}{2.6}=5$ & $78^{\circ} 41^{\prime}$ & $\frac{2.6}{13.0}=0.2$ & $11^{\circ} 18^{\prime}$ \\
\hline$\frac{26-2.0}{36.1-34.9}=\frac{24.0}{1.2}=20$ & $87^{\circ} 8^{\prime}$ & $\frac{1.2}{24.0}=0.05$ & $2^{\circ} 51^{\prime}$ \\
\hline$\frac{11-10.9}{33.5-29.0}=\frac{0.1}{4.5}=0.0222$ & $1^{0} 16^{\prime}$ & $\frac{4.5}{0.1}=45$ & $77^{\circ} 28^{\prime}$ \\
\hline$\frac{29-19.0}{36.6-32.2}=\frac{10.0}{4.4}=2.2727$ & $66^{\circ} 15^{\prime}$ & $\frac{4.4}{10.0}=0.44$ & $23^{\circ} 44^{\prime}$ \\
\hline$\frac{32-10.0}{36.2-29.1}=\frac{22.0}{7.1}=3.0985$ & $72^{\circ} 6^{\prime}$ & $\frac{7.1}{22.0}=0.3227$ & $17^{\circ} 53^{\prime}$ \\
\hline$\frac{56.0-45}{31.7-30}=\frac{11.0}{1.7}=6.4706$ & $81^{0} 12^{\prime}$ & $\frac{1.7}{11.0}=0.1545$ & $8^{\circ} 46^{\prime}$ \\
\hline$\frac{28.0-16.0}{33.4-28.5}=\frac{12.0}{4.9}=2.4489$ & $67^{\circ} 47^{\prime}$ & $\frac{4.9}{12.0}=0.4083$ & $22^{\circ} 12^{\prime}$ \\
\hline
\end{tabular}

te es de gran ayuda para el análisis e interpretación de los datos; basados en la propuesta del presente estudio, los resultados obtenidos se transformaron a partir de razones trigonométricas en ángulos tangente resultantes, lo cual ayuda a la interpretación objetiva del comportamiento entre las temperaturas analizadas y el tiempo de duración de la epilepsia, de esta manera, la inclinación angular está relacionada con el mayor valor del numerador respecto al denominador, por lo cual, el ángulo resultante tiende a $90^{\circ}$, tal y como se observa en la Tabla 2 .

Esto demuestra que el uso de la razón de cambio para el análisis de cuatro variables, dos relacionadas con el tiempo y dos con la temperatura registrada con base en áreas del cerebro, ayuda a la interpretación de las variables con respecto al mismo sujeto.

\section{CONCLUSIONES}

Los resultados demuestran la importancia de adaptar modelos matemáticos y aritméticos para el análisis e interpretación de las diferentes mediciones obtenidas en los pacientes con epilepsia farmacorresistente sometidos a cirugía. Las investigaciones con estas características son fundamentales, ya que, a partir de la aplicación de la muestra, se pueden construir tablas predictivas relacionadas con las variables de edad, tiempo con epilepsia y temperaturas cerebrales registradas para establecer velocidades de cambio a partir de los ángulos tangentes resultantes.

\section{BIBLIOGRAFÍA}

1. Beghi E, Giussani G, Sander JW. The natural history and prognosis of epilepsy. Epileptic Disord. 2015; 17 (3): 243-253.

2. https://www.who.int/es/news-room/detail/20-06-2019-whohighlights-scarcity-of-treatment-for-epilepsy-in-low-incomecountries.

3. Mbuba CKT, Ngugi AK, Newton CR, Carter JA. The epilepsy treatment gap in developing countries: a systematic review of the magnitude, causes, and intervention strategies. Epilepsia. 2008; 49 (9): 1491-1503.

4. Carrizosa J, Braga P, Albuquerque M, Bogacz A, Burneo J et al. Epilepsy for primary health care: a cost-effective Latin American E-learning initiative. Epileptic Disord. 2018; 20 (5): 386-395.

5. Andrade DM, Bassett AS, Bercovici E, Borlot F, Bui E, Camfield $\mathrm{P}$ et al. Epilepsy: Transition from pediatric to adult care. Recommendations of the Ontario epilepsy implementation task force. Epilepsia. 2017; 58 (9): 15021517.

6. De Font E, Terrazo J, López P, Collado MA, Díaz JL, Arch E et al. Thermography mapping patterns in temporal lobe epilepsy surgery. Surg Neurol Int. 2020; 11: 30. 